\title{
Commentary: Why use the Y-graft?
}

\author{
Ming-Sing Si, MD, Vikram Sood, MD, and Edward Bove, MD
}

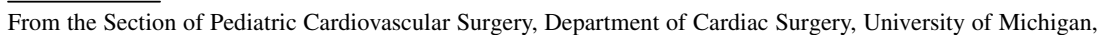
Ann Arbor, Mich.

Disclosures: Authors have nothing to disclose with regard to commercial support.

Received for publication Aug 27, 2019; accepted for publication Aug 28, 2019; available ahead of print Oct 23, 2019.

Address for reprints: Ming-Sing Si, MD, Section of Pediatric Cardiovascular Surgery, Department of Cardiac Surgery, 11-735 C.S. Mott Children's Hospital SPC 4204, 1540 E Hospital Dr, Ann Arbor, MI 48109-4204

(E-mail: mingsing@umich.edu).

J Thorac Cardiovasc Surg 2020;159:662-4

$0022-5223 / \$ 36.00$

Copyright $(2019$ by The American Association for Thoracic Surgery

https://doi.org/10.1016/j.jtcvs.2019.08.054

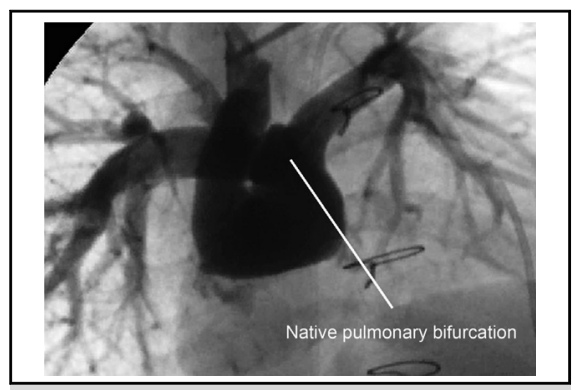

Native pulmonary bifurcation used in the hemi-Fontan and Fontan procedures.

\section{Central Message}

Use of the native pulmonary bifurcation can also be used to distribute flow and hepatic factor in the Fontan procedure.

See Article page 652
The Fontan procedure represents the last stage of surgical palliation for functional single-ventricle defects. As a staged operation in the current era, the Fontan procedure creates a pathway that directs inferior vena cava (IVC) blood flow into the lungs via the branch pulmonary arteries. Over time, a number of modifications of the Fontan procedure have been made, including an atriopulmonary connection, intra-atrial lateral tunnel, extracardiac conduit, and extracardiac Y-graft.

Two important long-term implications of a total cavopulmonary connection are hepatic factor distribution and total cavopulmonary resistance. Size and compliance of the Fontan pathway may also be important to the long-term outcomes. The lack of hepatic factor has been linked to the formation of arteriovenous malformations (AVMs) within the lungs, resulting in right-to-left shunting. ${ }^{1}$ Therefore, achieving bilateral pulmonary distribution of hepatic factor to both lungs is essential to avoid the development of AVMs. Total cavopulmonary resistance within the Fontan pathway is also of paramount importance, because pulmonary blood flow requires a low resistance pathway and passive flow. As such, pathways that minimize energy loss are believed to improve long-term single-ventricle palliation. ${ }^{2}$

Trusty and colleagues ${ }^{3}$ present midterm data on the use of a bifurcated Y-graft as a modification to the Fontan procedure for single-ventricle palliation. Although the data are limited by sample size (only 10 subjects) and there is no optimal control group, their findings suggest that use of a Y-graft promotes balanced hepatic flow distribution without an increase in total cavopulmonary connection resistance. The limited number of patients in this study combined with the comparisons of the Y-graft and conventional extracardiac conduit Fontan in the early postoperative period that demonstrated no difference or even worse hepatic flow distribution $^{4}$ perhaps tempered the enthusiasm and

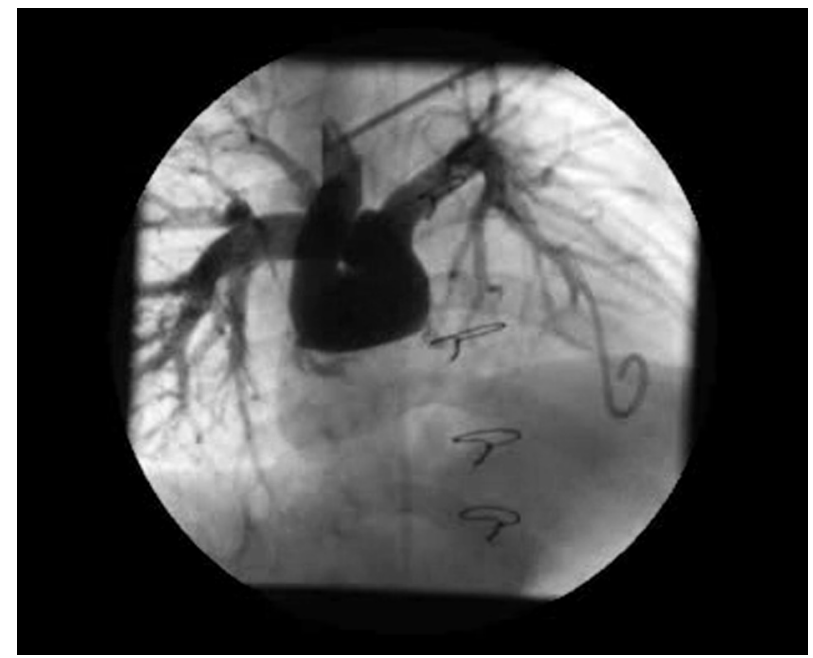

VIDEO 1. Anteroposterior view of a cineangiogram with contrast injection into the superior vena cava of a patient who underwent hemi-Fontan that demonstrates an intact cavoatrial junction, gentle U-turn of flow from inferior to superior and from right to left, and antegrade flow into the native pulmonary bifurcation as well as bilateral branch pulmonary arteries. Video available at: https://www.jtcvs.org/article/S0022-5223(19) 31806-9/fulltext. 


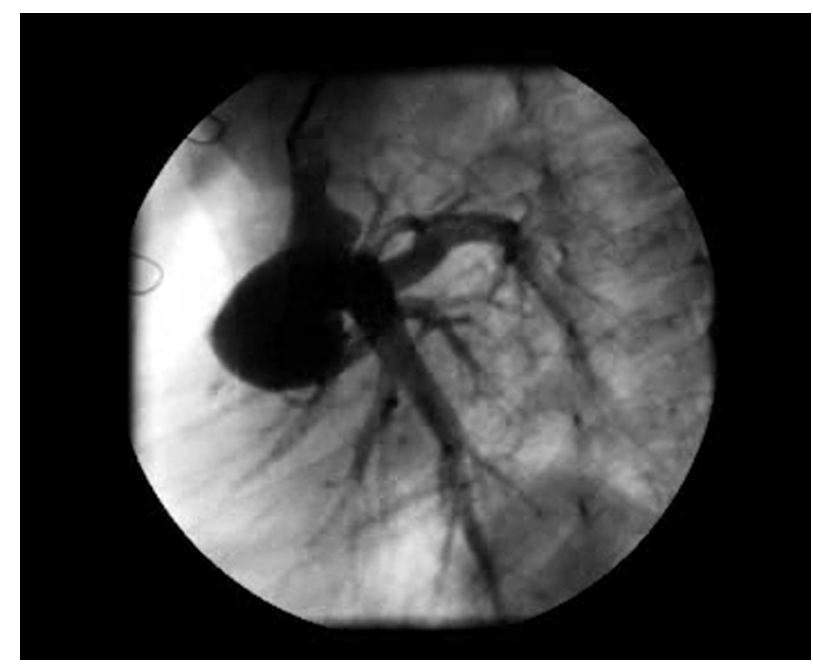

VIDEO 2. Lateral view of the same cineangiogram in Video 1. Note superior vena cava flow is initially posterior and then takes a gentle U-turn of flow from posterior to anterior and then antegrade into the native pulmonary bifurcation and branch pulmonary arteries. Video available at: https://www.jtcvs.org/article/S0022-5223(19)31806-9/fulltext.

widespread adoption of the Y-graft technique. In this midterm study, the investigators were able to demonstrate that the hepatic flow distribution improved over time with the Y-graft connection as well as the conventional extracardiac conduit, whereas total resistance remained unchanged over several years of follow-up.

The preference of the Y-graft geometry over a T-type connection achieved in an end-to-side anastomosis with a nonbifurcated extracardiac conduit is intuitive. However, from a practical standpoint, routine use of this approach may be difficult - as the investigators note in their discussion-because of insufficient space to appropriately bevel 2 separate conduits. Furthermore, each limb of the Y-graft accommodates less flow and therefore raises concern for risk of thrombosis. Nonetheless, the Y-graft technique may be considered as a potential option for treating patients who have developed pulmonary AVMs after more conventional types of Fontan procedures.

At the University of Michigan, we have utilized a lateral tunnel Fontan pathway for the majority of our singleventricle patients. Although there are different techniques to create a lateral tunnel Fontan, our approach utilizes the native pulmonary bifurcation, thereby preserving a natural pulmonary arterial flow distribution to both lungs. ${ }^{5}$ Accomplishing the Michigan lateral tunnel is best performed by utilizing a hemi-Fontan procedure rather than a bidirectional Glenn at the prior volume unloading stage of reconstruction. During the hemi-Fontan, superior vena cava flow is channeled through the atrium and atrial appendage, leaving the cavoatrial junction intact. ${ }^{5}$ After the completion of surgical reconstruction with a lateral tunnel, IVC flow is then directed into the main pulmonary artery and split with the native bifurcation (Videos 1 and 2), while avoiding right angle connections. Significant advantages of this technique include offset of the caval flows in the anterior-posterior plane to prevent collision and energy $\operatorname{loss}^{6}$ (Figure 1), growth potential, a compliant wall in contradistinction to a stiff synthetic graft, and equal distribution of IVC flow to both lungs. When anatomic variations of pulmonary and systemic venous connections preclude the use of this

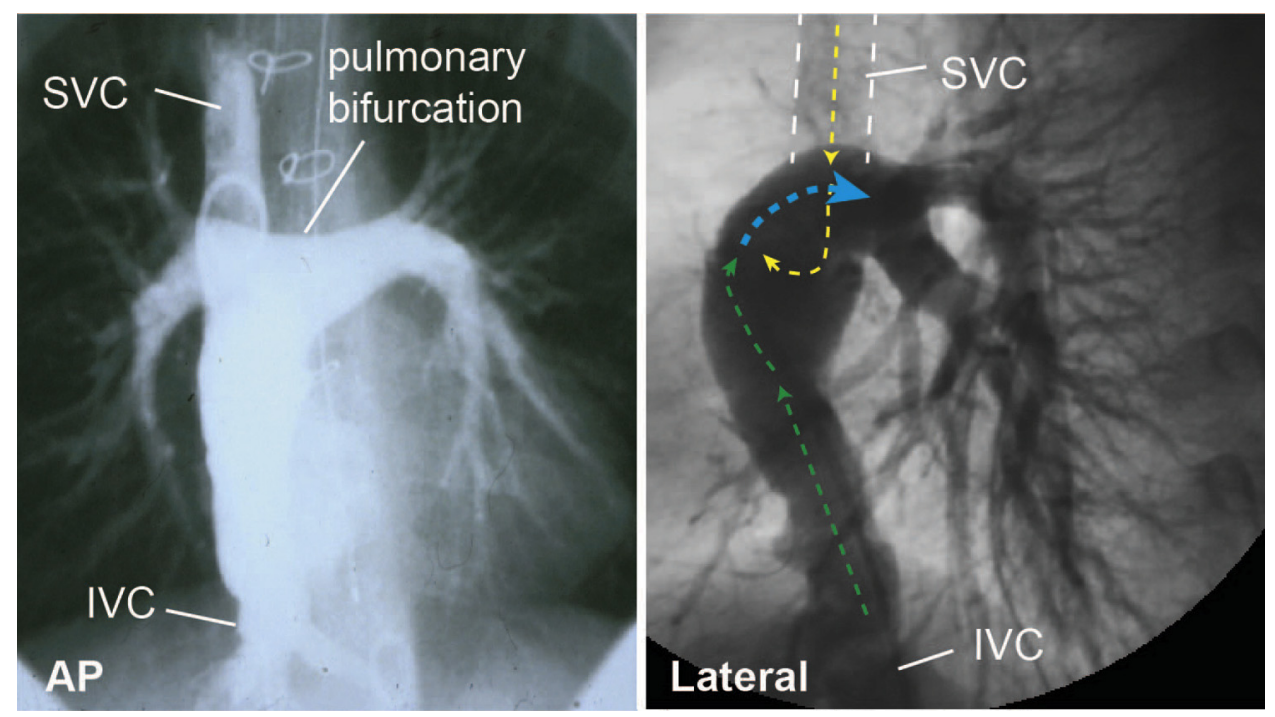

FIGURE 1. Anteroposterior $(A P)$ and lateral angiogram images of a Michigan lateral tunnel Fontan. Injection of contrast was performed in the inferior vena cava $(I V C)$. Lateral view demonstrates anterior/posterior offset of flow from the superior vena cava (SVC) (yellow) and IVC (green) into the branch pulmonary arteries. Combined systemic venous flow (blue), representing the entire cardiac output, then passes antegrade through the native pulmonary bifurcation. 
approach, we employ an extracardiac conduit that is anastomosed to the superior vena cava above the bidirectional Glenn to promote venous mixing before entrance into the branch pulmonary arteries.

The Fontan procedure and its subsequent variations represent a major landmark in the management of patients with single-ventricle defects and was made possible by the ingenuity and courage of pioneering surgeons and patients. Although some of these variations of the Fontan may perform reasonably well in the first and second decades of life, it may take several more decades before differences are observed. Perhaps future generations of surgeons will know whether utilizing the native pulmonary bifurcation, a product of millions of years of evolution, is better, equivalent, or worse than nonbifurcated or bifurcated synthetic grafts in the Fontan circulation.

\section{References}

1. Srivastava D, Preminger T, Lock JE, Mandell V, Keane JF, Mayer JE Jr, et al. Hepatic venous blood and the development of pulmonary arteriovenous malformations in congenital heart disease. Circulation. 1995;92:1217-22.

2. Murakami H, Yoshimura N, Kitahara J, Otaka S, Ichida F, Misaki T. Collision of the caval flows caused early failure of the Fontan circulation. J Thorac Cardiovasc Surg. 2006;132:1235-6.

3. Trusty PM, Wei Z, Sales M, Kanter KR, Fogel MA, Yoganathan AP, et al. Y-graft modification to the Fontan procedure: increasingly balanced flow over time. J Thorac Cardiovasc Surg. 2020;159:652-61.

4. Trusty PM, Restrepo M, Kanter KR, Yoganathan AP, Fogel MA, Slesnick TC. A pulsatile hemodynamic evaluation of the commercially available bifurcated Y-graft Fontan modification and comparison with the lateral tunnel and extracardiac conduits. J Thorac Cardiovasc Surg. 2016;151:1529-36.

5. Hirsch JC, Ohye RG, Devaney EJ, Goldberg CS, Bove EL. The lateral tunnel Fontan procedure for hypoplastic left heart syndrome: results of 100 consecutive patients. Pediatr Cardiol. 2007;28:426-32.

6. Bove EL, de Leval MR, Migliavacca F, Guadagni G, Dubini G. Computational fluid dynamics in the evaluation of hemodynamic performance of cavopulmonary connections after the Norwood procedure for hypoplastic left heart syndrome. J Thorac Cardiovasc Surg. 2003;126:1040-7. 